\title{
Comparison of two computerized procedures for the assessment of color discrimination in Cebus apella
}

\author{
Sheila T. Makiama, Paulo R. K. Goulart and Olavo F. Galvão \\ Universidade Federal do Pará, Belém, PA, Brazil
}

\begin{abstract}
Color vision consists of the discrimination of objects based on their spectral composition. Among primates, the majority of Platyrrhini monkeys are estimated to have polymorphic and sex-linked dichromacy. The objective of this study was to compare the results produced by different equipment and software for the assessment of tri- and dichromatic conditions in one male and two female Cebus apella. Three experiments were programmed. In Experiment 1, verifying the trichromatic condition of one female subject and dichromatic condition of the remainder of the subjects was possible using an adapted version of the Cambridge Colour Test. Experiment 2 confirmed the results of Experiment 1 using a different array of stimuli of the same test. Experiment 3, which produced results similar to Experiment 2, consisted of a test developed for a standard computer system using stimuli with color properties similar to the ones used in the previous experiment. Favorable conditions for the assessment of color vision in Platyrrhini can be built with low-cost equipment and software. Once data have been gathered with additional subjects and new stimulus arrangements have been tested and confirmed, the procedure can be used for the evaluation of other Platyrrhini species for which behavioral color discrimination data are currently lacking. Keywords: color vision, Cambridge Colour Test, Platyrrhini, Cebus apella.
\end{abstract}

Received 3 June 2011; received in revised form 27 September 2011; accepted 27 September 2011. Available online 29 December 2011

\section{Introduction}

The perception of color depends on the presence of at least two classes of cone photoreceptors in the retina, each with distinct but overlapping spectral sensitivity (TessierLavigne, 1991; Surridge, Osorio, \& Mundy, 2003). Among primates, the genotype/phenotype arrangement called regular trichromacy, based on the presence of three classes of cone photoreceptors with each class maximally sensitive to short, medium, and long wavelength light, is exhibited by humans, Old World (Catarrhini) primates (Jacobs \& Hartwerth, 1989; Dulai, von Dornum, Mollon, \& Hunt, 1999), and at least one species of New World (Platyrrhini) monkey (i.e., the howler monkey Allouata sp.; Jacobs, Neitz, Deegan, \& Neitz, 1996). Although the shortwavelength (S)-sensitive photoreceptor is encoded by a gene located on an autosomal chromosome, in these species medium/long-wavelength (M/L)-sensitive photoreceptors are encoded by two genes located at different loci on the X chromosome (Hunt et al., 1998; Surridge et al., 2003).

Color vision in most Platyrrhini monkeys studied so far is characterized by a sex-linked polymorphism

Makiama, S.T., Goulart, P.R.K., and Galvão, O.F., Núcleo de Teoria e Pesquisa do Comportamento, Universidade Federal do Pará. Correspondence regarding this article should be directed to: Sheila Makiama, Trav. Mauriti, 970, apt. 105, Belém, PA, Brazil, CEP 66080-650. Telephone: +55-91-88170896. E-mail: kildare12001@yahoo.com.br.
(Jacobs, 1984; Jacobs, Neitz, \& Neitz, 1993; Jacobs, 1996) attributable to the production of $\mathrm{M} / \mathrm{L}$ photoreceptors determined by allelic versions of a gene located at a single locus on the X chromosome (Jacobs \& Neitz, 1987; Jacobs, 1996). Electrophysiological studies performed with squirrel monkeys (Saimiri sciureus), a traditional model of Platyrrhini color vision, have found that multiple alleles for the gene responsible for $\mathrm{M} / \mathrm{L}$ photoreceptors correlated with the production of receptors with slightly different spectral sensitivities (Jacobs, 1998; Jacobs \& Deegan, 2003). These findings were later extended for other Platirrhini species, including capuchin monkeys (Cebus sp.; Jacobs, \& Deegan, 2003), for which at least three functionally different $\mathrm{M} / \mathrm{L}$ cone photopigments have been confirmed. Because males have a single $\mathrm{X}$ chromosome, each individual can express only one of three possible $\mathrm{M} / \mathrm{L}$ cone photoreceptors that, combined with the $\mathrm{S}$ photoreceptor, produce one of three dichromatic conditions. The same outcome is seen in homozygous females (i.e., those showing the same allele in both of their X chromosomes). Heterozygous females, in contrast, express two different $\mathrm{M} / \mathrm{L}$ photoreceptors along with the S photoreceptor, consequently showing one of three types of trichromacy (Jacobs, 1996).

The scenario outlined above appears to be the norm among Platirrhini species. The only two exceptions known so far are owl monkeys (Aotus sp.), which have a 
monochromatic condition attributable to the presence of only one cone type (Jacobs, Deegan, Neitz, Crognale, \& Neitz, 1993), and the aforementioned howler monkeys (Alouatta sp.), for which electrophysiological examinations of the retina discovered a trichromatic system similar to the one found in Old World monkeys (Jacobs et al., 1996).

\section{Cambridge Colour Test}

The Cambridge Colour Test (CCT; Mollon \& Reffin, 1989; Regan, Reffin, \& Mollon, 1994; Mollon \& Regan, 2000) is a computer-controlled color discrimination test that uses pseudoisochromatic stimuli to control spatial and luminance cues. The test displays an array of circles that vary in size (spatial noise) and luminance (luminance noise), in which a target is differentiated from the background only by differences in chromaticity (hue and/or saturation). One advantage of the computerized control of this test is that the chromaticity difference between the target and background can be varied dynamically and adaptively according to the performance of the participant.

The general structure of the test is the following. The background is set with a chromaticity value usually represented as a point in the CIE 1931 or CIE 1976 chromaticity diagram, and the target is defined at another point in the chromaticity diagram (i.e., with a different hue). The line that links these points forms a vector along which the distance between target-background is varied depending on the performance of the participant. Discrimination thresholds were obtained by averaging the distances from the background point of the hues in which correct and incorrect responses occurred. The distribution of threshold points at critical regions of the chromaticity diagram is used to diagnose color vision variations (Regan et al., 1994).

Although the CCT was originally developed and validated for adult human observers, the test was recently adapted for use with squirrel monkeys (Saimiri sp.) by Mancuso, Neitz, \& Neitz (2006), producing results that were consistent with the dichromatic condition of two male monkeys as predicted by DNA analysis and electroretinography examination. These results validated the CCT as a tool for evaluating color discrimination in nonhuman primates. A similar adaptation made for human children (Goulart, Bandeira, Tsubota, Oiwa, Costa, \& Ventura, 2008) is currently used with capuchin monkeys in our laboratory. Despite being based on a chromaticity diagram derived from human observers, the test is sensitive to the color vision variations of both squirrel and capuchin monkeys because of the similarities between the spectral sensitivities of the cone photoreceptors present in those species and those found in humans (Mancuso et al., 2006; Jacobs \& Deegan, 2003). In both adaptations, a critical modification was the change in the shape of the target stimulus from a Landolt-C to a "square" that could appear at different locations on the monitor screen and should be touched by the subjects.
The reliable behavioral segregation of the capuchin monkeys' color vision in its trichromatic/dichromatic subtypes requires costly high-precision software and equipment. The optimal hardware for the CCT includes a high-performance, heavy 22 inch CRT monitor and graphic card for stimulus generation and display. In this context, developing a reliable, low-cost, and lighter alternative for reliable color vision assessment in nonhuman primates would provide conditions for Platirrhini color vision assessment with modest investment compared with CCT costs.

Goulart, Makiama, Fonseca, Marques \& Galvão (2008) evaluated the color discrimination of two capuchins, a male and a female, through successive sessions of simple discrimination using a regular PC and monitor. The hues that served as stimuli were produced using the Paint application for Microsoft Windows XP. In each session, a pair of hues was presented. The trials consisted of 16 squares presented in a $4 \times 4$ matrix on the monitor screen, one $(\mathrm{S}+)$ defined with a hue different from the other 15 (S-). In the beginning of the training, no luminance noise was present, so the correct selection could be prompted by intensity cues. In subsequent sessions, when the subjects were consistently selecting the $\mathrm{S}+$, every 16 stimuli were randomly defined with different values of luminance and saturation. In this condition, the subjects failed to discriminate between the yellow-red, yellow-green, and green-red pairs, showing that although the stimuli used in that study were produced and displayed using low-precision software, monitor, and graphic card, the participants showed performance that was consistent with theoretical predictions for the species. A vulnerability of that study was that critical test pairs were composed of arbitrarily chosen hues from the general color categories usually said to be confused by dichromatic individuals. Despite the consistent performance with the generic test pairs, a more precise control of the hues was necessary to confirm the viability of this low-cost setting to evaluate color discrimination.

The present study sought to replicate the findings previously obtained with the low-cost equipment using a new set of more precisely defined hues as stimuli and verify the results against measures performed with the same hues using the high-precision apparatus (i.e., CCT). If the apparatus and behavioral procedures adopted in the present study are valid, then they may be used for the preliminary assessment of color vision in other Platyrrhini species for which behavioral data are scarce, such as the howler monkey (Allouata sp.).

\section{General methods}

Three experiments with different objectives were conducted. Experiment 1 sought to determine the color vision phenotype of each subject using the CCT. Experiment 2 sought to investigate the indications of trichromacy/ 
dichromacy subtypes found in the previous procedure using different chromaticities in the CCT apparatus. Experiment 3 proposed to replicate the results of Experiment 2 using a standard computer system. Differences in procedures and stimuli are described in each section below. The subjects and apparatus are described below.

\section{Subjects}

Three capuchin monkeys (Cebus cf. apella), one male (M1) and two females (F1 and F2), participated in this study. All subjects were proficient in performing simple discrimination tasks on a touch-sensitive screen. The subjects were kept with three other animals of the same species in separate cages $(2.5 \times 2.5 \times 2.5$ $\mathrm{m})$ partially covered by a roof $(2.0 \times 2.5 \mathrm{~m})$. The procedures adopted over the course of the experiment were approved by the Ethics Committee for Animal Research, Federal University of Pará (license no. CEPAE-UFPA PS001/2005) and were in accordance with local and international rules on the treatment and manipulation of animals for research purposes. The subjects were fed once per day in the afternoon, and their diet consisted of a special ration for primates, including fruits, vegetables, and vitamin supplements. Water was available ad libitum.

\section{Apparatus}

An experimental chamber that measured $60 \times 60 \times$ $60 \mathrm{~cm}$ with three walls made of transparent Plexiglas and one wall made of aluminum was used in Experiments 1 and 2 (Figure 1A). The aluminum wall had a $40 \mathrm{x}$ $30 \mathrm{~cm}$ opening, framed by a wooden structure used to adjust the distance (up to a maximum length of 26 $\mathrm{cm}$ ) between the chamber and a 22 inch CRT monitor (Mitsubishi Diamond Pro 2070SB). Just above the door of the chamber was a mini camera directed to the monitor screen. A CarrolTouch infrared touch interface was attached to the end of the wooden structure. The touch interface consisted of a grid of infrared lightemitting diodes (LEDs), which was used to identify the position of the touches on the monitor screen. A pellet dispenser (MED Associates, Inc.) adapted to the top outside the chamber delivered $190 \mathrm{mg}$ banana pellets to a small open box above the monitor.

The control of the experimental parameters in Experiments 1 and 2 was performed by a Dell Precision 670 microprocessor running a version of the CCT adapted by Marcio L. Bandeira, Paulo R.K. Goulart, Nestor N. Oiwa, Marcelo Costa, and Dora F. Ventura in Object Pascal language (Borland's Delphi 7.0; used by Goulart et al., 2008). Stimuli were generated by the ViSaGe System (Cambridge Research Systems), which produced a target-background arrangement characteristic of the adapted version of the CCT, with a square with $5 \mathrm{~cm}$ sides as the target stimulus that could be presented at nine different positions on the CRT monitor
(Figure 1B). The CRT was calibrated by a ColorCal photometer (Cambridge Research Systems) through the VSG Desktop library (version 8.0) calibration routine.

A $60 \times 60 \times 60 \mathrm{~cm}$ experimental chamber with three walls made of transparent acrylic and one wall made of aluminum was used in Experiment 3 in which a rectangular window gave access to a touch-sensitive LCD monitor (1928L 19 inch ELO Touchmonitor, Tyco Electronics; Figure 1C). The stimulus display presented 25 squares $(5 \times 4 \mathrm{~cm}$ each) in a $5 \times 5$ matrix on a black background (Figure 1D). All stimuli in Experiment 3 were produced by a freeware image editor (Gimp 2.6; The Gimp Team). Experimental manipulations were performed using the custom-made software PCR (Portuguese acronym for Reinforcement Contingencies Programming, developed by Marcio L. Bandeira, Paulo R.K. Goulart, Romariz S. Barros, and Carlos B. de Sousa) running on an Intel Core 2 Duo processor with a 2 GB Windows XP Professional operating system, version 2002, and standard graphics card.

\section{Experiment 1}

\section{Method}

\section{Stimuli}

The stimuli were presented in the target-background arrangement characteristic of the adapted version of the CCT. For the training sessions, the target and background were defined at points that were not part of any predicted confusion line for the dichromatic phenotypes expected for capuchins. Depending on the training session, the background could assume the coordinates $\mathrm{u}^{\prime}=0.167$ and

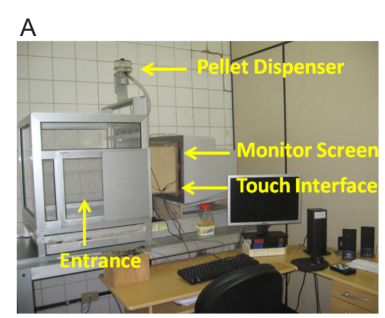

C
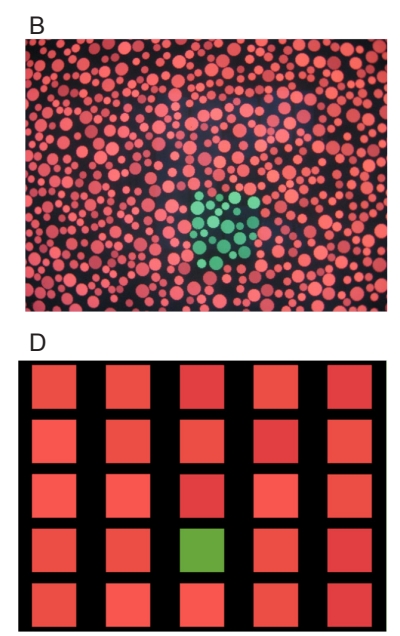

Figure 1. Experimental chamber and stimuli presented in Experiments 1 and 2 (A and B, respectively) and experimental chamber and stimuli presented in Experiment 3 (C and D, respectively). In Experiments 1 and 2, the array of stimuli was presented in the adapted version of the Cambridge Colour Test, and in Experiment 3, the arrangement of stimuli was programmed by PCR and reproduced using a standard computer system. 
Table 1. The u'v' coordinates of each stimulus used in the test sessions of Experiments 1 and 2

\begin{tabular}{cccccccccccc}
\hline & \multicolumn{3}{c}{ Stimuli of Experiment 1 } & \multicolumn{9}{c}{ Stimuli of Experiment 2 } \\
\hline Points & Achromatic & Protan & Deutan & Tritan & Green1 & Green2 & Red1 & Red2 & Red3 & Red4 & Blue \\
\hline u' & 0.1888 & 0.678 & -1.271 & 0.2573 & 0.135 & 0.136 & 0.377 & 0.362 & 0.281 & 0.311 & 0.171 \\
v' & 0.4607 & 0.501 & 0.782 & 0.0 & 0.545 & 0.504 & 0.517 & 0.482 & 0.526 & 0.486 & 0.228 \\
\hline
\end{tabular}

$v^{\prime}=0.444$ (gray), and targets could be u' $=0.171$ and $v^{\prime}=0.228$ (blue) or $\mathrm{u}^{\prime}=0.184$ and $\mathrm{v}^{\prime}=0.556$ (yellow). The background could also assume the coordinates $u^{\prime}=$ 0.171 and $v^{\prime}=0.228$ (blue), and the target could be u' $=$ 0.184 and $v^{\prime}=0.556$ (yellow). For the test session, the background was set at the achromatic point of the CIE 1976 chromaticity diagram, and the hue of the target was defined along three vectors that corresponded to the "protan," "deutan," and "tritan" test vectors. The protan and deutan points were the same as those used by Mancuso et al. (2006) in their adaptation of the CCT for use in nonhuman subjects. The tritan coordinates were the same as those used as default in the CCT. The u'v' coordinates that defined each vector are presented in Table 1 (left).

\section{Procedure}

Before the test sessions, training sessions were conducted to guarantee that the subjects would keep selecting the target even when the target-background chromatic difference was dim. The number of training sessions varied according to the performance of each subject. In the beginning of each session, the target chromaticity was defined at the most distant point of a given vector (i.e., with the maximum contrast against the background). The maximum distance between the target and background depended on the distance of the points in the CIE 1976 diagram, which was calculated using the formula $x^{2}+y^{2}=$ $z^{2}$, where $x=$ distance, $y=u^{\prime} \mathrm{a}-\mathrm{u}$ 'b, and $z=\mathrm{v}^{\prime} \mathrm{a}-\mathrm{v}$ 'b.

Initially, the target-background minimum distance was $0.05\left(500 \times 10^{-4}\right)$ points in the CIE 1976 diagram. When the subjects were consistently responding to the target even at low contrast, the minimum distance was reduced to $0.02\left(200 \times 10^{-4}\right)$.

During the sessions, the chromaticity difference between target and background increased or decreased depending on the performance of the subject. Correct responses produced a decrease in the distance in the next trial. Incorrect responses produced an increase in the target-background distance in the next trial. The number of trials in a session depended on the performance of the subject. The duration of each trial was $10 \mathrm{~s}$, and the intertrial interval (ITI) was $6 \mathrm{~s}$. A touch on the target ended the trial with the release of a $190 \mathrm{mg}$ bananaflavored pellet and the beginning of the ITI. Touches at another position were counted as errors, producing the end of the trial and the beginning of the ITI. The criterion for ending each training session was three consecutive correct trials with the minimum target- background distance for each axis of the session.

The general structure of the test sessions was similar to the training sessions. The difference was that three new vectors were tested, consisting of hues that were predicted to serve as confusion lines for human dichromatic phenotypes. Each subject was exposed to two test sessions, 1 week apart. The test presented the protan, deutan, and tritan vectors. The target-background maximum distance was set at $0.11\left(1100 \times 10^{-4}\right)$ points, and the minimum distance required for all participants was $0.002\left(20 \times 10^{-4}\right)$ points in the diagram.

The criterion for the end of the test sessions was 11 reversals (error followed by a hit or hit followed by an error) in each axis, after which the software automatically calculated thresholds of color discrimination from the average of the distances of the last seven reversals. The thresholds were presented as (average distance) x $10^{-4}$ units u'v'.

\section{Results and discussion}

All subjects were exposed to the test sessions only after reaching the acquisition criterion in the training phase. Figure 2 presents the average thresholds obtained by subjects F1, F2, and M1 at the protan, deutan, and tritan test vectors. These vectors are intended to present the subjects with hue discriminations that would be difficult or impossible for each one of the three human dichromatic conditions: protanopia, deuteranopia, and tritanopia. Because the dichromatic phenotypes presented by capuchin monkeys are similar to the protan and deutan human dichromacies, at least according to the receptors involved, the male monkey was expected to fail at the protan or deutan

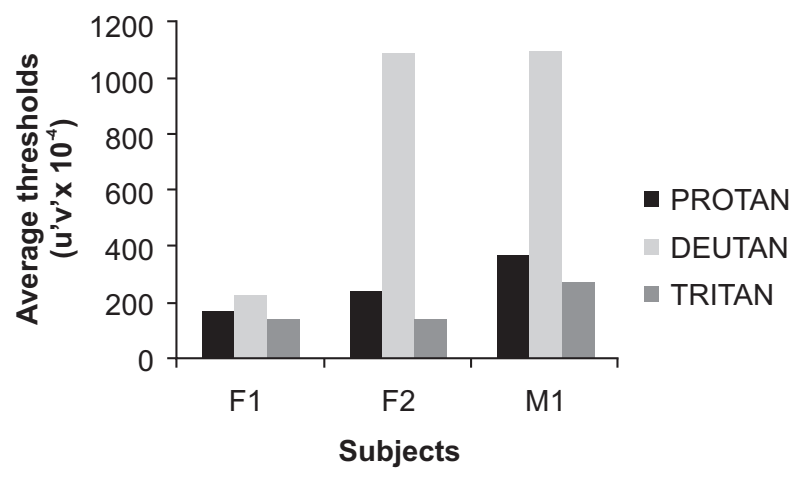

Figure 2. Average threshold of subjects F1, F2, and M1 in test session of Experiment 1. 
vectors. The females could fail at both of these vectors or perform as trichromats.

The male and female F2 exhibited thresholds of

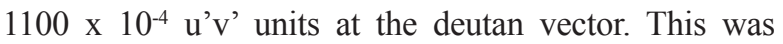
the maximum distance between the background-target chromaticity points in this test. The discrimination thresholds obtained for the other vector were near the lower limit (0.02) established during the training phase. These results were consistent with a dichromatic condition based on the presence of a medium/long wavelengthsensitive photopigment with peak sensitivity close to $560 \mathrm{~nm}$. The other female, F1, showed thresholds near the baseline lower limit for all test vectors, performance consistent with a trichromatic condition.

Experiment 2 sought to further investigate the indications of trichromacy (F1) and deutan-like dichromacy (F2 and M1) using different chromaticities as background. The target-background pairs were chosen to coincide with protan and deutan confusion lines plotted on the CIE 1976 chromaticity diagram. If the performance of all of the participants with these new hues was consistent with the phenotypes suggested by the results of Experiment 1, then the same chromaticity values would be used in Experiment 3.

\section{Experiment 2}

\section{Method}

\section{Stimuli}

The stimuli included four long-wavelength hues (Red1, Red2, Red3, and Red4), two medium-wavelength hues (Green1 and Green2), and one short-wavelength hue (Blue). The coordinates of each stimulus are shown in Table 1 (right), and their positions in the diagram are shown in Figure 3.

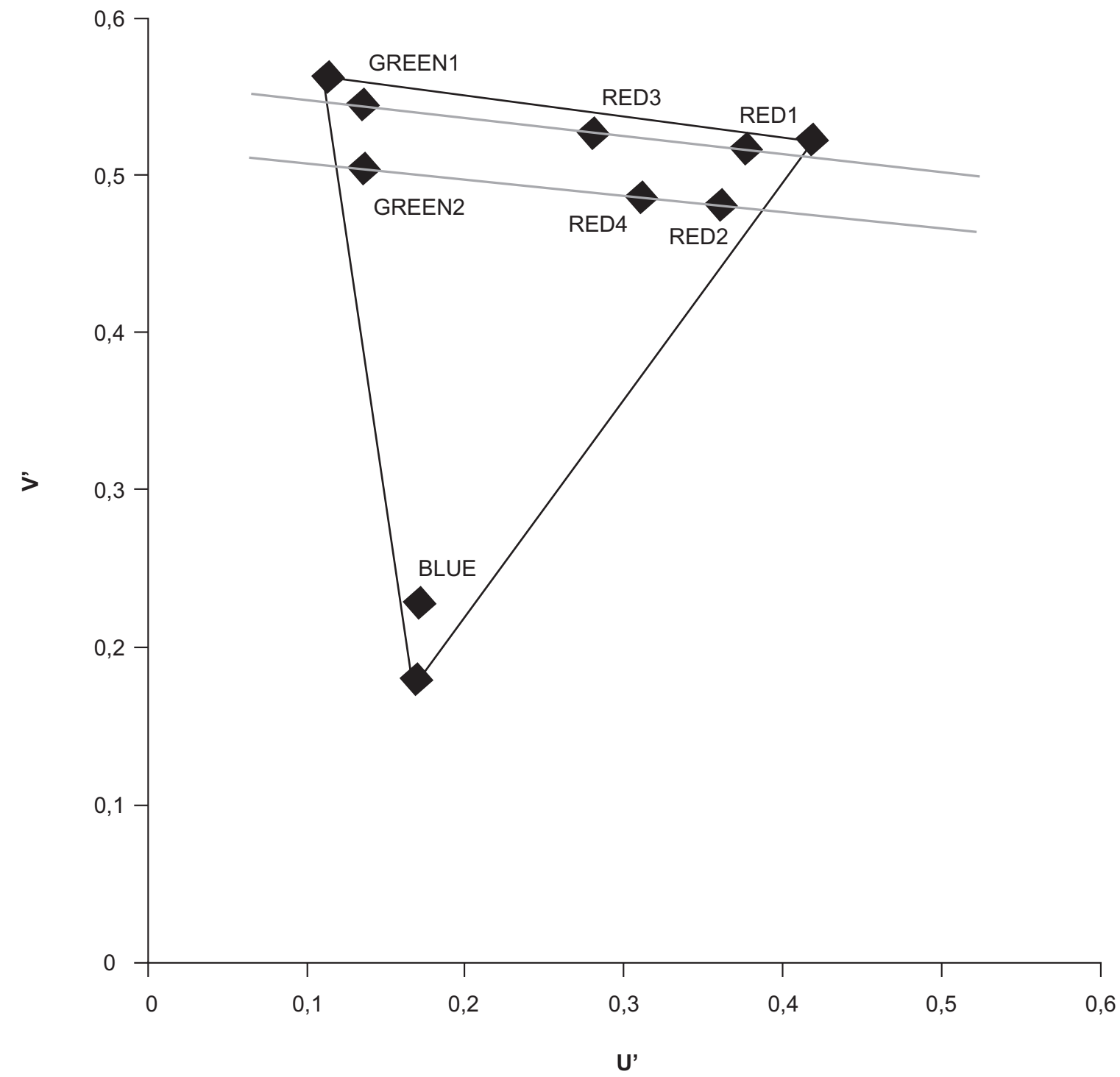

Figure 3. Graphic representation of position of points in the CIE 1976 chromaticity diagram used in Experiment 2: u' $=0.135$ and $v^{\prime}=0.545($ Green 1$), u^{\prime}=0.377$ and $v^{\prime}=0.517(\operatorname{Red} 1), u^{\prime}=0.281$ and $v^{\prime}=0.526(\operatorname{Red} 3)$ connected by a straight line, $u^{\prime}=$ 0.136 and $v^{\prime}=0.504\left(\right.$ Green2), $u^{\prime}=0.362$ and $v^{\prime}=0.482(\operatorname{Red} 2), u^{\prime}=0.311$ and $v^{\prime}=0.486(\operatorname{Red} 4)$ also connected by a straight line, and $\mathrm{u}^{\prime}=0.171$ and $\mathrm{v}^{\prime}=0.228$ (Blue). 
Table 2. Test session schedule of Experiment 2

\begin{tabular}{lllll}
\hline & Test 1 & Test 2 & Test 3 & Test 4 \\
\hline Background & Red1 & Red2 & Red3 & Red4 \\
Axis 1 & Green1 & Green1 & Green1 & Green1 \\
Axis 2 & Green2 & Green2 & Green2 & Green2 \\
Axis 3 & Blue & Blue & Blue & Blue \\
\hline
\end{tabular}

Table 3. Maximum distance between target and background in the 1976 CIE chromaticity diagram

\begin{tabular}{lllllllll}
\hline $\begin{array}{l}\text { Pair of } \\
\text { stimuli }\end{array}$ & $\begin{array}{l}\text { Green1- } \\
\text { Red1 }\end{array}$ & $\begin{array}{l}\text { Green1- } \\
\text { Red2 }\end{array}$ & $\begin{array}{l}\text { Green1- } \\
\text { Red3 }\end{array}$ & $\begin{array}{l}\text { Green1- } \\
\text { Red4 }\end{array}$ & $\begin{array}{l}\text { Green2- } \\
\text { Red1 }\end{array}$ & $\begin{array}{l}\text { Green2- } \\
\text { Red2 }\end{array}$ & $\begin{array}{l}\text { Green2- } \\
\text { Red3 }\end{array}$ & $\begin{array}{l}\text { Green2- } \\
\text { Red4 }\end{array}$ \\
\hline $\begin{array}{l}\text { Maximum } \\
\text { distance }\end{array}$ & 0.244 & 0.236 & 0.147 & 0.186 & 0.242 & 0.228 & 0.147 & 0.176 \\
\hline
\end{tabular}

\section{Procedure}

Each participant was exposed twice to four test sessions. The second round of tests was performed 1 week after the first round. Tests 1 and 2 were designed to present discriminations that involved pairings of long-wavelength hues (Red1 and Red2) and mediumwavelength hues (Green1 and Green2) that would be confusing for dichromatic subjects. Tests 3 and 4 sought to verify the results obtained from Tests 1 and 2 by presenting as background new stimuli (Red3 and Red4) chosen from confusion lines suggested by the subjects' performance in the previous tests. The target-background arrangement of all four test sessions is shown in Table 2. Trials with the Blue target were inserted in each session to serve as a "measure of responsiveness." Because the discrimination in those trials was based on short-wavelength components, they did not constitute a challenge to the most common dichromatic conditions, characterized by insensitivity to medium/long wavelengths.

The target-background maximum distance depended on the distance between the corresponding points in the chromaticity diagram. The maximum distance of each pair of stimuli is shown in Table 3 .

Thresholds were considered "normal" when they were near or below $200 \times 10^{-4}$, the minimum distance required in the training sessions. A given pair of hues was considered a confusion pair for a given subject when the animal presented thresholds above $500 \times 10^{-4}$. If the subject failed to select the target in the maximum distance for five consecutive trials, then the threshold was defined at its maximum value, which corresponded to the maximum distance between target-background.

\section{Results and discussion}

Figure 4 shows the discrimination thresholds from Tests 1 to 4 . In Test 1 (Figure 4A), subjects F2 and M1 presented thresholds of $1314 \times 10^{-4}$ and $1418 \times 10^{-4}$, respectively, for the Green1-Red1 (target-background) discrimination, characterizing the arrangementas difficult to discriminate for both subjects. In Green2-Red1 trials in this test, subjects F2 and M1 presented thresholds of $575 \times 10^{-4}$ and $234 \times 10^{-4}$, respectively. Subject F1 presented low thresholds in both discriminations: 210 x $10^{-4}$ in Green1- Red1 trials and $184 \times 10^{-4}$ in Green2Red1 trials. In Test 2 (Figure 4B), all of the subjects presented thresholds above $500 \times 10^{-4}$ for the Green 1 Red2 (target-background) discrimination, characterizing this arrangement as difficult to discriminate for all subjects. In the Green2-Red2 discrimination, subject F1 presented a threshold of $385 \times 10^{-4}$, and subjects F2 and M1 presented thresholds of $1484 \times 10^{-4}$ and $1228 \times 10^{-4}$, respectively, characterizing this arrangement as difficult to discriminate for both subjects.

The results from Tests 1 and 2 identified two pairs of hues confused by subjects M1 and F2 (Green1-Red1 and Green2-Red2). Tests 3 and 4 then sought to verify these results by using novel background hues from the confusion lines that contained those pairs. The results of Tests 3 and 4 are shown in Figures 4C and 4D, respectively. Subjects M1 and F2 presented thresholds above $500 \times 10^{-4}$ for both the Green 1-Red3 (Test 3 ) and Green2-Red4 (Test 4) vectors, confirming the confusion lines predicted from the previous tests.

All of the subjects presented thresholds below 500 $\mathrm{x} 10^{-4}$ in trials that involved the short-wavelength hue target (Blue), indicating that the subjects continued to consistently respond to the chromatic difference even when it was very subtle. This indicates that the high thresholds obtained during the tests were indeed attributable to low detectability rather than general performance degeneration.

Subject F1 exhibited low discrimination thresholds for all test vectors, except in the Green1-Red2 vector in Test 2. The general performance of subject F1 in Experiment 2 corroborates the trichromatic color vision phenotype indicated in Experiment 1. The thresholds presented by subjects M1 and F2 for the critical test vectors were also consistent with the dichromatic color vision indicated in Experiment 1. Figure 3 shows the 

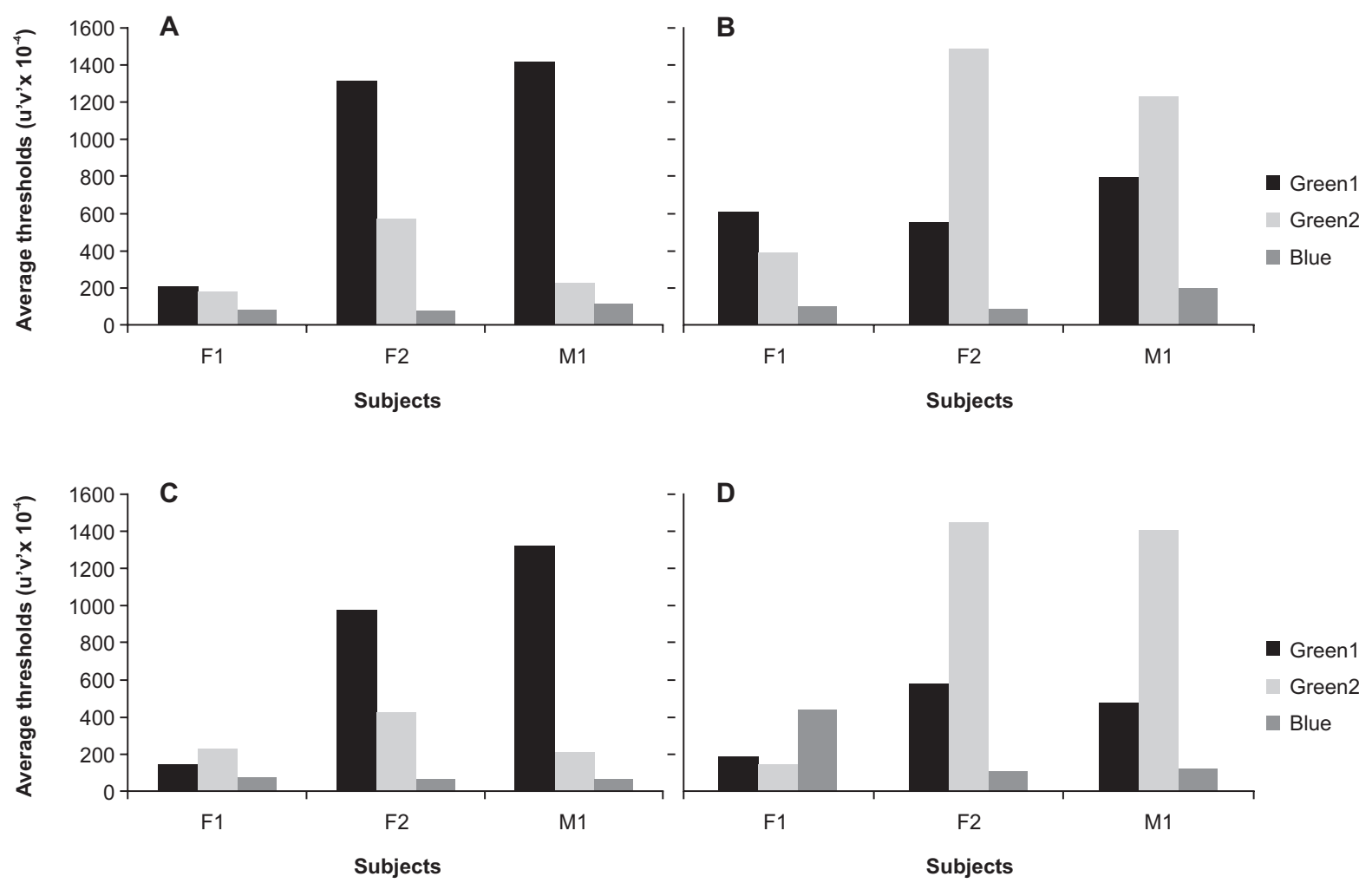

Figure 4. Average thresholds for subjects F1, F2, and M1 in test sessions 1 to 4. The targets in all four tests were Green1, Green2, and Blue. The background in Test 1 (A) was Red1, in Test 2 (B) was Red2, in Test 3 (C) was Red3, and in Test 4 (D) was Red4. See text for details.

chromaticity coordinates of the seven test hues in the CIE 1976 chromaticity diagram and the confusion lines inferred from the subjects' performance, one containing the Green 1, Red3, and Red1 confusion points and the other containing the Green2, Red4, and Red2 confusion points. The orientation of the lines in the CIE 1976 diagram is consistent with the confusion lines characteristic of the human deuteranope condition, a result consistent with the data collected from Experiment 1.

Experiment 2 identified pairs of long and medium wavelength hues that are confusing for some dichromatic subjects. Experiment 3 used the same stimuli to assess the viability of evaluating color discrimination using standard equipment and image-editing software.

\section{Experiment 3}

\section{Method}

\section{Stimuli}

Four hues (Green1, Red1, Red3, and Blue) were defined at the same u'v' coordinates used in Experiment 2. The hues were measured on the LCD monitor screen with a ColorCal photometer using the LightScan application (Cambridge Research Systems). Each hue could be presented at one of five luminance levels: 20 , $25,30,35$, and $40 \mathrm{~cd} / \mathrm{m}^{2}$.

\section{Procedure}

The number of trials was fixed, regardless of the subject's performance. In both the baseline and test sessions, each trial presented one square defined with a particular hue $(\mathrm{S}+)$ and the other 24 squares defined with a second hue (S-), which could appear in any of the luminance variations previously mentioned. The squares that presented the $\mathrm{S}+$ and different luminance levels of the S- were randomly assigned and different from trial to trial. Selecting the $\mathrm{S}+$ produced a $190 \mathrm{mg}$ banana pellet and the end of the trial. Selecting any of the S- stimuli produced only the end of the trial. The ITI was $5 \mathrm{~s}$, and the duration of the trial depended on the occurrence of a touch response anywhere on the screen. The criterion for ending the sessions of this experiment was the conclusion of all trials or 5 min elapsing without any touches on the screen.

Before being presented with the test sessions, each subject was exposed to two baseline sessions with 20 trials each. The first session presented Blue as the $\mathrm{S}+$ and Red1 as the $\mathrm{S}-$. The second session presented Blue as the $\mathrm{S}+$ and Red 3 as the $\mathrm{S}$-. In both cases, the luminance of the $\mathrm{S}+$ was fixed $\left(20 \mathrm{~cd} / \mathrm{m}^{2}\right)$, whereas the luminance of the S- varied between $20,25,30,35$, and $40 \mathrm{~cd} / \mathrm{m}^{2}$. The criterion for the end of this phase was $70 \%$ or more correct responses.

After reaching the acquisition criterion, each subject was exposed to two test sessions with 40 trials each, with 
16 trials that presented baseline discriminations and 24 trials that presented test discriminations. The test stimuli were selected based on the results of Experiment 2. In the first session, the test trials were composed of Green $1(\mathrm{~S}+)$ and Red1 (S-). Baseline trials were composed of Blue (S+) and Red1 (S-). In the second session, the test trials were composed of Green1 (S+) and Red3 (S-). Baseline trials were composed of Blue (S+) and Red3 (S-).

\section{Results and discussion}

All three participants achieved the learning criterion of $70 \%$ or more correct responses in both baseline sessions (Fig. 5A). In test sessions 1 and 2 (Figures $5 \mathrm{~B}$ and $5 \mathrm{C}$, respectively), all three subjects maintained good and consistent performance in the baseline trials. The first test session evaluated Green1-Red1 discrimination (Fig. 5B). Only subject F1 consistently selected the S+ $87.5 \%$ accuracy). Subjects F2 and M1 selected the $\mathrm{S}+$ in $12.5 \%$ and $18.75 \%$ of the test trials, respectively. In this procedure, the percentage of responses that corresponded to chance was near $6.25 \%$. Therefore, this performance was interpreted as reflecting difficult (but not impossible) discrimination,

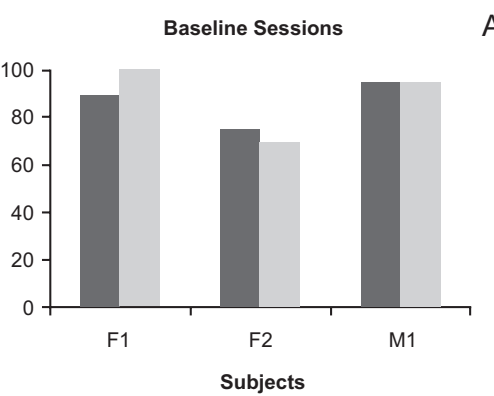

A
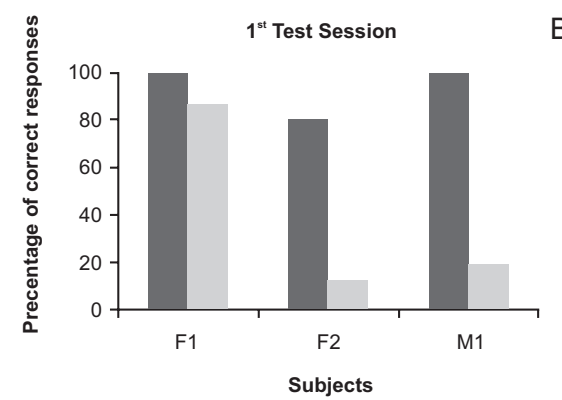

B

Baseline Session1 (Blue-Red1)

Baseline Session2 (Blue-Red3)

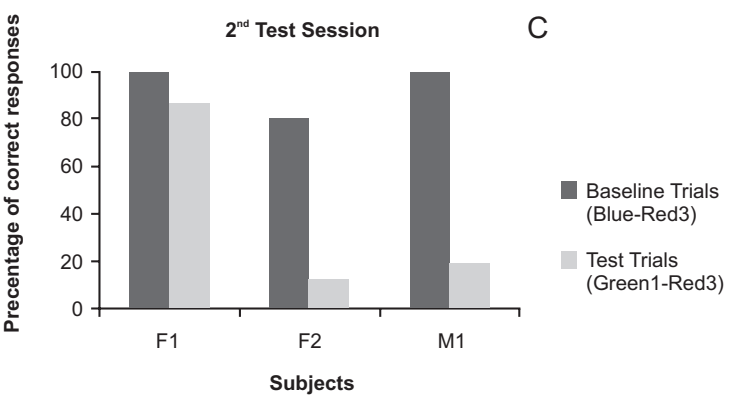

Figure 5. Percentage of correct responses under baseline sessions (A), in the first test session (B), and in the second test session (C) of Experiment 3. probably because of the fact that the test points did not coincide exactly with a dichromatic confusion line. The second test session evaluated the discrimination Green1Red3 (Figure 5C). As expected, because Red3 was in the same confusion line predicted from the Green1-Red1 discrimination, subject F1 performed with high accuracy $(87.5 \%)$, whereas subjects F2 and M1 showed nearchance performance $(8.3 \%$ and $0 \%$, respectively). These results indicate that Green1-Red3 posed an impossible discrimination for the dichromatic subjects.

These results are consistent with those obtained in Experiment 2, in which the discriminations Green1Red1 and Green1-Red3 were tested in a different arrangement with a standard computer system. The performance of subject F1 was consistent with the trichromacy in Experiment 2 (Figure 4) and Experiment 3 (Figure 5). The performance of subjects F2 and M1 were indicative of dichromacy in both experiments.

The coherence between the results from Experiments 2 and 3 suggests that favorable conditions for the evaluation of color vision in individuals of the genus Cebus sp. can be created with hardware and software with low financial cost. Future studies could extend the present investigation with a larger number of subjects and sessions that involve other stimuli in which the subjects have previously presented high discriminative thresholds, such as in arrangements that involve Green2, Red2, and Red4.

\section{General discussion}

This study demonstrated the feasibility of the investigation of color vision in capuchin monkeys when applying two different arrangements of equipment and procedures that differ in their technical standards and cost. Experiments 1 and 2 confirmed the applicability of the study in capuchin monkeys using the CCT. The adapted version of the Mollon-Reffin test, based on the version used by Goulart et al. (2008), was able to produce challenging arrangements for the dichromatic phenotype in capuchin monkeys and provided evidence of the specific form of dichromacy. The effectiveness of a similar adaptation with nonverbal subjects has been demonstrated in Saimiri sp. using the test version of Mancuso et al. (2006). The modifications in software, equipment, and training techniques produced suitable conditions for the test application and allowed the acquisition and maintenance of responding in non-human primates under the control of the task contingencies. The need for verbal instruction was bypassed by the requirement of a touch on a monitor screen and the adoption of operant conditioning techniques, making the demand of an answer consistent with the phylogenetic characteristics of the species in this study. The results are also consistent with the scientific literature, which predicts the sex-linked polymorphism of color vision among individuals of the genus Cebus. 
The phenotypes inferred from the behavioral tests in the experiments reported here were later confirmed in a study performed in parallel by Goulart et al. (2011), in which 10 capuchin monkeys, including subjects M1, F1, and F2, had their DNA analyzed and were tested with the adapted version of the CCT for 20 test vectors around three background hues in the CIE 1976 u'v' diagram. The genetic sequencing allowed the deduction of the amino acid combination at positions 180, 277, and 285 of the resulting opsins, from which the absorption peak of the corresponding photopigments was inferred (based on Soares et al., 2010). Female F1 was identified as a trichromat, presenting the amino acid combinations adenine-phenylalanine-adenine (AFA) and adeninephenylalanine-threonine (AFT), corresponding to peaks at $532 \mathrm{~nm}$ and $542-547 \mathrm{~nm}$, respectively. Subjects M1 and F2 were identified as deuteranope dichromats, with an absorption peak at $560-563 \mathrm{~nm}$, inferred from the amino acid combination serine-thyrosine-threonine (SYT).

Experiment 3 provided some evidence that favorable conditions for the evaluation of color vision in capuchin monkeys can be built using a low-cost, standard computer system because creating situations in which the difference in hue is the only property consistently correlated with reinforcement is possible. Thus, although the monitor and video card used were less than those considered optimal for the generation and control of chromatic stimuli, reproducing a challenging arrangement for the dichromatic subjects identified in Experiments 1 and 2 was possible. This was possible by creating an arrangement of multiple stimuli with the same shape but varied luminance so that the stimulus that differed in hue could be identified only based on this property.

Compared with the ViSaGe system, which automatically controls hue and luminance variations, the low-cost solution proposed here presents the experimenter with the additional task of calculating and producing, with the help of a colorimeter attached to the monitor, every variation of hue and luminance necessary for the test. Additionally, if the test is eventually to be used with a monitor different from the one for which the stimuli were originally calibrated, correcting the stimulus values individually for the specific monitor will be necessary. Another advantage of the CCT that should be noted is the possibility of dynamic variation of the target-background chromatic difference. These qualities make the CCT the ideal choice for color vision testing whenever possible. The simplified system presented here enables the assessment of color discrimination when operating and financial costs would make the use of the CCT unavailable. The demonstration in this study of favorable conditions for the application of a color vision test with Cebus indicates that the evaluation of tri- and dichromacy in other platyrrhine primates for whom data are scarce or do not exist may also be performed with the system.

\section{Acknowledgments}

A previous version of this paper was presented as the Master's Thesis of S.T. Makiama. This research was funded by FINEP/FADESP (Ref. 4191/05, Proc. no. 01.06.0842-00, Conv. 1723, IBN Net) and CNPq P620226/2008-0, P481438/2008-4, and INCT-ECCE. Support for this research was provided by the National Council for Scientific and Technologic Development (CNPq) to S.T. Makiama (Master's Degree Fellowship), P.R.K. Goulart (Postdoctoral Fellowship), and O.F. Galvão (Research Productivity Fellowship). Additional support was provided by the Brazilian Ministry of Technology in the program of the National Institutes of Science and Technology on Behavior, Cognition, and Teaching (Program Announcement 15/2008, CNPq grant no. 573972/2008-7, FAPESP grant no. 2008/57705-8).

\section{References}

Dulai, K. S., von Dornum, M., Mollon, J. D., \& Hunt, D. M. (1999). The evolution of trichromatic color vision by opsin gene duplication in New World and Old World primates. Genome Research, 9, 629-638.

Goulart, P. R. K., Makiama, S. T., Fonseca, A. R., Marques, S., K. L, \& Galvão, O.F. (2008). Visão de cores em Cebus apella: Avaliação de discriminação de cores por meio de um monitor CRT padrão e ferramenta de edição de cores do Windows XP. Neurociências, 4(2), 80-86.

Goulart, P. R. K., Bandeira, M. L., Tsubota, D., Oiwa, N. N., Costa, M. F., \& Ventura, D. F. (2008). A computer-controlled color vision test for children based on the Cambridge Colour Test. Visual Neuroscience, 25, 445-450.

Goulart, P. R. K., Bonci, D. M. O., Mesquita, C. S., Pereira, F. S., Costa. J. R., Borges, R. P., Leal, T. R. C., Galvão, O. F., Silveira, L. C. L., \& Ventura, D. F. (2011). Colour discrimination in the Capuchin monkey, Cebus apella. Manuscript in preparation.

Hunt, D. M., Dulai, K. S., Cowing, J. A., Julliot, C., Mollon, J. D., Bowmaker, J. K., Li, W. H., \& Hewett-Emmett, D. (1998). Molecular evolution of trichromacy in primates. Vision Research, 38, 3299-3306.

Jacobs, G. H. (1984). Within-species variations in visual capacity among squirrel monkeys (Saimiri sciureus): Color vision. Vision Research, 24, 1267-1277.

Jacobs, G. H. (1996). Primate photopigment and primate color vision. Proceedings of the National Academy of Sciences of the United States of America, 93, 577-581.

Jacobs, G. H. (1998). A perspective on color vision in platyrrhine monkeys. Vision Research, 38, 3307-3313.

Jacobs, G. H., \& Deegan, J. F., 2nd (2003). Cone pigment variations in four genera of new world monkeys. Vision Research, 43, 227-236.

Jacobs, G. H., Deegan, J. F., 2nd, Neitz, J., Crognale, M. A., \& Neitz, M. (1993). Photopigment and color vision in nocturnal monkey, Aotus. Vision Research, 33, 1773-1783.

Jacobs, G. H., \& Hartwerth, R. S. (1989). Color vision variation in Old and New World primates. American Journal of Primatology, $18,35-44$.

Jacobs, G. H., \& Neitz, J. (1987). Inheritance of color vision in a New World monkey (Saimiri sciureus). Proceedings of the National Academy of Sciences of the United States of America, 84, 2545-2549.

Jacobs, G. H., Neitz, J., \& Neitz, M. (1993). Genetic basis of polymorphism in the color vision of Platyrrhine monkeys. Vision Research, 33, 269-274.

Jacobs, G. H., Neitz, M., Deegan, J. F., \& Neitz J. (1996). Trichromatic color vision in New World monkeys. Nature, 382, 156-158.

Mancuso, K., Neitz, M., \& Neitz, J. (2006). An adaptation of the Cambridge Colour Test for use with animals. Visual Neuroscience, 23, 695-701

Mollon, J. D., \& Reffin, J. P. (1989). A computer-controlled colour vision test that combines the principles of Chibret and Stilling. 
Journal of Physiology, 414, 5P.

Mollon, J. D., \& Regan, B. C. (2000). Cambridge Color Tes Handbook [http://visl.technion.ac.il/projects/2002w/theory.pdf accessed September 29, 2011].

Regan, B. C., Reffin, J. P., \& Mollon, J. D. (1994). Luminance noise and the rapid determination of discrimination ellipses in colour deficiency. Vision Research, 34, 1279-1299.

Soares, J. G., Fiorani, M., Araújo, E. A., Zana, Y., Bonci, D. M. O., Neitz, M., Ventura, D.F., \& Gattass, R. (2010). Cone photopigment variations in Cebus apella monkeys evidenced by electroretinogram measurements and genetic analysis. Vision Research, 50, 99-106.

Surridge, A. K., Osorio, D., \& Mundy, N. I. (2003). Evolution and selection of trichromatic color vision in primates. Trends in Ecology and Evolution, 18, 198-205.

Tessier-Lavigne, M. (1991). Phototransduction and information processing in the retina. In E. R. Kandel, J. H. Schwartz, \& T. M. Jessel (Eds.). Principles of neural science, 3rd edition (pp. 400416). Norwalk, CT: Appleton \& Lange. 